\title{
Identifying the Youngest Proto Planetary Nebulae
}

\author{
B. M. Lewis ${ }^{1}$ \\ ${ }^{1}$ Arecibo Observatory, HC3 Box 53995, Arecibo PR00612, USA \\ email: blewis@naic.edu
}

\begin{abstract}
Detached shells can be identified from their NIR \& MIR colours. We use this to identify PPN candidates, including three like IRAS $18455+0448$. These are $19178+1206,18470+0015$ $\& 18123+0511$.
\end{abstract}

Keywords. stars: AGB and post-AGB

The PPN phase begins when $\mathrm{dM} / \mathrm{dt} \rightarrow 0$ permanently, so the first diagnostics are the loss of LPV variability, and the presence of a detached shell. Time and radial evolution of the fossil shell add exceptionally red MIR colors and a B to K spectral type to the list of indicators. Since past searches for PPN depend on these "secondary", evolvedstate, criteria, they did not identify IRAS $18455+0448$ as a young PPN, because it has a relatively blue (25-12) $\mu m=-0.275$. This object had a classic $1612 \mathrm{MHz} \mathrm{OH} / \mathrm{IR}$ star spectrum when discovered that has since died (Lewis, Oppenheimer \& Daubar 2001). Nevertheless it has an IRAS variability flag, var, that indicates a zero probability of it being a MIR variable, and its still extant $\mathrm{OH}$ mainline masers have a constant intensity. IRAS $18455+0448$ is our prototype for a young PPN from a low-mass progenitor star.

Post AGB stars with detached shells are readily identified by their NIR colours (Lewis, Kopon \& Terzian 2004), so we report here the results of a search for more young proto planetary nebulae in the Arecibo set of OH/IR stars with reliable 2MASS colours, (25-12) $\mu m>-0.4$, and var $<30 \%$. This results in the 25 objects highlighted in figure 1 , with the known planetary and proto planetary nebulae distinguished from the new objects, which are listed in Table 1. These scatter along the blue end of the NIR locus of OH/IR stars, with more dispersion than its other members. This is expected. If we assume a constant SED for the central star, simulations show that the NIR colors of a detached shell evolve along the locus toward its blue end (Lewis 2006), though if the SED evolves towards a B to K type, the corresponding NIR colors fall a little below the locus, and evolve parallel to it. Indeed it is clear from the group of known proto planetary nebulae at its blue terminus that these have evolved past the rest of the $\mathrm{OH} / \mathrm{IR}$ stars to the notably bluer NIR colors of the stellar SED.

Our list of 25 includes IRC +10420 , a hypergiant star with a detached shell that is unlikely to be a PPN. It also includes $19177+1333 \& 19261+1849$ that may be normal $\mathrm{OH} / \mathrm{IR}$ stars with small var due to the particular epochs at which IRAS observed them, or else very young PPN. The new candidate PPN most like $18455+0448$ is $19178+1206$, which has lost its weak $1612 \mathrm{MHz}$ feature, but still has a double-peaked $1667 \mathrm{MHz}$ feature. A second method of identifying a young PPN from a low mass progenitor is to check the list for objects that have an expansion velocity $V_{e}<12 \mathrm{~km} \mathrm{~s}^{-1}$, as suggested by studies of the Arecibo OH/IR stars (Lewis, Eder \& Terzian 1990). This highlights 18470+0015 as a red MIR star with bluer NIR colours than $18455+0448$, and a $V_{e}=4 \mathrm{~km} \mathrm{~s}^{-1}$, and 18123+0511, as likely new PPN. Both fall below the NIR locus. 
Table 1. New Candidate PPN from the Arecibo Set of OH/IR Stars

\begin{tabular}{cccccrl}
\hline IRAS & H-K & J-H & $(25-12) \mu m$ & $(60-25) \mu m$ & var \% & note \\
\hline $18123+0511$ & 0.670 & 0.575 & -0.394 & -0.842 & 5 & \\
$18455+0448$ & 1.989 & 1.774 & -0.275 & -0.783 & 0 & PPN \\
$18470+0015$ & 1.689 & 1.618 & 0.009 & -0.531 & 15 & \\
$19177+1333$ & 2.509 & 2.718 & -0.112 & -0.880 & 4 & OH/IR? \\
$19178+1206^{*}$ & 0.728 & 1.965 & -0.038 & -0.672 & 1 & \\
$19227+1700$ & 1.199 & 2.289 & -0.227 & -1.039 & 12 & \\
$19261+1849$ & 2.593 & 4.055 & -0.259 & -0.748 & 9 & OH/IR? \\
$19319+2214$ & 1.256 & 1.665 & -0.091 & -0.742 & 3 & \\
$19520+2759$ & 1.607 & 2.053 & -0.003 & -0.175 & 16 & \\
\hline
\end{tabular}

* no $1612 \mathrm{MHz}$ emission now; var \% 三 IRAS probability of being a MIR variable

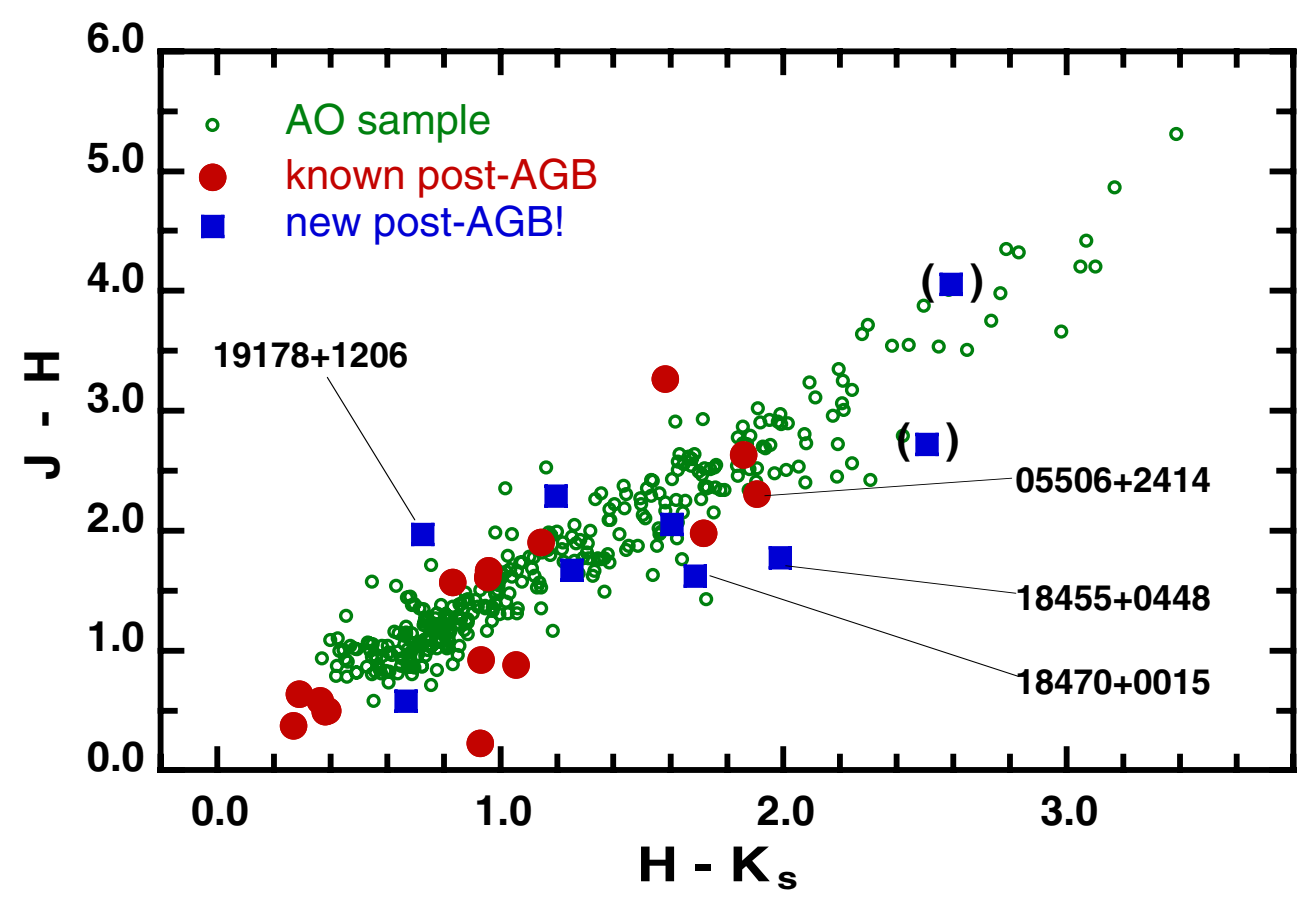

Figure 1. The 2MASS two-colour plot of Arecibo OH/IR stars with reliable fluxes. Those with an IRAS variability flag var $<30 \%$ of being MIR variables are marked with filled symbols: $\bigcirc$ for known PN \& PPN, $\square$ for new candidates.

\section{Acknowledgements}

This work is supported by the National Astronomy and Ionosphere Center, which is operated by Cornell University under a cooperative agreement with the National Science Foundation.

\section{References}

Lewis, B. M., Eder, J., \& Terzian, Y. 1990, ApJ 362, 634

Lewis, B. M., Oppenheimer, B. D. \& Daubar, I. J. 2001, ApJ (Letters) 548, L77

Lewis, B. M., Kopon, D. A., \& Terzian, Y. 2004, AJ 127, 501

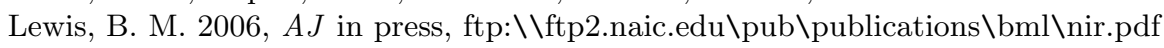

